\title{
CONTENIDO, SENSACIÓN Y PERCEPCIÓN
}

\author{
Olga Fernández Prat \\ Departamento de Filosofía \\ Facultad de Filosofía y Letras \\ Universitat Autònoma de Barcelona \\ olga.fernandez@uab.cat
}

RESUMEN: Este artículo pretende clarificar la relación entre los aspectos sensoriales de la percepción y el aspecto "demostrativo" sobre el que han llamado la atención autores como Evans y McDowell. Tras introducir un análisis husserliano en espíritu de los modos de presentación demostrativo-perceptuales, y con base en una versión de la teoría de los datos sensoriales — sin los defectos que la han hecho impopular recientemente entre los filósofos - se proponen correspondencias entre la "fase sensorial" y la "fase demostrativo-conceptual" de la percepción, caracterizadas, respectivamente por su contenido no conceptual y conceptual. El punto crucial de la relación entre ambos se explica entonces apelando a cierta operación sintética. Finalmente, se intenta mostrar que esta propuesta es más satisfactoria que la propuesta alternativa existente, debida a John Campbell.

PALABRAS CLAVE: datos sensoriales, modos de presentación, contenido demostrativo

SUMMARY: This paper aims at clarifying the relationship between the sensory aspects and the "demonstrative" aspect of perception that has attracted the interest of philosophers like Evans and McDowell. On the basis of a broadly Husserlian analysis of perceptual demonstrative modes of presentation, on the one hand and, a recent version of the sense-data theory of perception on the other — without the drawbacks that have tended to render that theory impopular among philosophers - correspondences between the "sensory phase" and the "demonstrative and conceptual phase" of perception are proposed, once those "phases" have been characterized, respectively, by non-conceptual and conceptual contents. The key point of the relationship between these is then explained by appealing to a certain synthetic operation. Finally, an attempt is made to show that this is a more satisfactory proposal than the alternative by John Campbell.

KEY WORDS: sense data, modes of presentation, demonstrative content

Cuando percibimos un objeto en una ocasión cotidiana en que ese objeto nos concierne (justo vemos que un libro que buscábamos está sobre la mesa, pongamos por ejemplo), ese objeto se nos hace presente de un modo especial, de un modo que, de momento, sólo acertamos a expresar demostrativamente ("Ése es el libro que buscaba"). Este, denominémoslo así, "aspecto demostrativo" de la percepción ha llamado recientemente la atención de un buen número de destacados filósofos en la tradición analítica (Evans, Searle, McDowell, Burge, Brewer y Campbell, entre otros). Sin embargo, fue en la tradición 
fenomenológica, y muy especialmente en Husserl, donde primero se llamó la atención sobre el mismo y donde primero se intentó su elaboración teórica. El primer objetivo del presente trabajo es, justamente, exponer de forma breve el tratamiento husserliano de esta cuestión. Como se verá, esta perspectiva conduce de un modo natural a abordar el problema de la relación entre sensación y percepción y, dentro de esta problemática general, el tema más específico de la relación entre el contenido no conceptual y el contenido conceptual de la percepción. El propósito principal de este trabajo es contribuir a explicar esta relación, para lo cual habrán de especificarse previamente los dos términos de la misma.

La sección 1 se dedica a explicar el mencionado "aspecto demostrativo" en el contexto de la teoría husserliana de la percepción, el cual, como se verá, presenta un interés no meramente histórico y facilita la identificación de los problemas que hace falta resolver. En la sección 2 se expone un análisis de los modos de presentación demostrativo-perceptuales que, por las razones que allí se apuntarán, es, según creo, husserliano en espíritu, pero se diferencia de otros análisis en la misma línea, como los de Searle y Woodruf Smith, sobre los que ofrece ventajas. Husserl distinguió en un estado perceptual ${ }^{1}$ un componente cercano - digámoslo de momento vagamente - a los datos sensoriales postulados por las teorías empiristas, por lo que surge la cuestión de la relación entre ese componente y el carácter demostrativo de la percepción, cuestión sobre la que el propio Husserl (pace Føllesdal 1976) no ofreció una solución adecuada. Pues bien, justamente es una versión reciente de una teoría de los datos sensoriales la que se toma como uno de los términos - contenido no conceptual - de la relación que se pretende explicar, y la sección 3 se dedica a presentar esa versión y mostrar sus ventajas con respecto a teorías de los datos sensoriales más tradicionales. En la sección 4 se argumenta brevemente que, supuesta esa versión de la teoría, los datos sensoriales pueden considerarse, efectivamente, como contenidos no conceptuales, y se plantea el problema específico de la relación entre sensación y percepción sobre el cual aquí se intenta dar una solución como el problema de especificar, en el marco del análisis de la percepción, la correspondencia entre dos tipos diferentes de contenido, el contenido no conceptual, representado por los datos

\footnotetext{
${ }^{1}$ Utilizo el término "perceptual" para referirme no sólo a casos genuinos de percepción (percepción exitosa), sino también a casos en que se generan ilusiones o alucinaciones, dado que la expresión "estado de percepción" probablemente se interpretaría como aplicable sólo a los primeros.
} 
sensoriales, y el contenido conceptual, representado por los modos de presentación demostrativo-perceptuales y/o los juicios de los que éstos forman parte. Dirigida a la solución del problema así precisado, se una propuesta basada en cierta noción de síntesis de los elementos de los datos sensoriales y en la noción de atención. En la última sección se critica una propuesta reciente debida a John Campbell que quizá superficialmente podría considerarse similar a la aquí propugnada. Se muestran los rasgos que diferencian a ambas propuestas y se argumenta en detalle que el intrincado argumento con el que Campbell pretende salvar el principal escollo de su propuesta resulta basarse, en último término, en un uso equívoco del término "justificación". Finalmente se formulan algunas consideraciones adicionales a favor de la propuesta aquí propugnada.

\section{El carácter demostrativo de la percepción en el contexto de la teoría husserliana}

En los últimos años, diversos autores de la tradición analítica han insistido en que la percepción tiene, al menos frecuentemente, un carácter demostrativo y han tratado de proporcionar una explicación filosófica de en qué consiste ese rasgo central de la percepción. Sin embargo, Husserl, el fundador de la corriente fenomenológica, se había anticipado a identificar dicho rasgo, el cual se pone especialmente de relieve - de manera en general independiente de los autores aludidos de la tradición analítica- en intérpretes relativamente recientes como D.W. Smith, I. Miller y J. Lad (cfr. Smith 1984 y Miller 1984). ${ }^{2}$

Según Husserl, el aspecto demostrativo caracteriza todos los estados de percepción de objetos particulares. Consideremos, por ejemplo, una experiencia perceptual que quizá pudiéramos vernos inclinados a describir mediante el enunciado "Veo un pájaro negro". De acuerdo con lo dicho, una manera más reveladora de describir la experiencia en cuestión desde una perspectiva husserliana la proporcionaría el enunciado "Veo eso como un pájaro negro" (cfr. Smith

${ }^{2}$ En las Investigaciones Husserl presentó un análisis del significado y la referencia de los nombres propios y los pronombres demostrativos que difiere radicalmente del modelo descriptivista fregeano (cfr. Investigaciones lógicas, I, § 26, y VI, §§ 15 ; en adelante $L I$ ) y coincide con el punto de vista ampliamente compartido en la actualidad desde los trabajos de Kripke sobre los primeros y Kaplan sobre los segundos en sostener que ambos tipos de expresiones refieren "directamente", no a través de propiedades que el referente posea de modo único. Lo distintivo de Husserl es que sus reflexiones sobre la relación entre esa perspectiva semántica y el contenido intencional de los estados mentales se inscriben en una teoría general de la intencionalidad (véase Smith 1984). 
y McIntyre 1982, p. 214). Con esto se pretende poner de manifiesto que, para Husserl, un estado perceptual (un "acto" de percepción en la terminología husserliana) nos pone en una relación de inmediatez o "contacto directo" (acquaintance, en la conocida terminología russelliana) con un objeto. Sin embargo, incluso los enunciados del segundo tipo están, por decirlo así, a un grado de distancia de la experiencia perceptual. Lo que un enunciado así intenta describir es, en realidad, el juicio de percepción que podríamos hacer sobre la base de la experiencia perceptual, no propiamente esta experiencia. En efecto, Husserl considera y rechaza la posibilidad de que una expresión demostrativa utilizada en un contexto de percepción sirva simplemente para expresar lingüísticamente el contenido (o parte del contenido) de la experiencia perceptual misma ( $L I \mathrm{VI}, \S \S 3-4) .^{3}$

De acuerdo con la concepción husserliana, el aspecto demostrativo del contenido de la experiencia perceptual debe explicarse independientemente de la explicación del significado de las expresiones demostrativas, aunque no sea cierta la inversa. Con esto nos enfrentamos a la siguiente pregunta: ¿cómo articular teóricamente aquello en lo que consiste la inmediatez de nuestro rapport con el objeto?

Para explicar lo que pueda haber en Husserl de respuesta a esta pregunta necesitamos recordar que, para él, todo estado ("acto") intencional tiene - hablando preteóricamente - la característica de ser como si estuviese dirigido a un objeto, y que Husserl explica teóricamente esta característica como la posesión, por parte del acto, de un noema, noción que podemos considerar, en cierto modo, como una generalización —al dominio de los estados mentales intencionalesde la noción fregeana de sentido. ${ }^{4}$ Ahora bien, hay en la concepción de los noemata por parte de Husserl un aspecto que los diferencia decisivamente de los sentidos en la concepción estrictamente fregeana de los mismos (por contraposición a algunas concepciones neofregeanas) y que se pone de manifiesto especialmente en relación con las experiencias perceptuales. Un noema (en particular, el noema correspondiente a una experiencia perceptual, que es lo que aquí nos interesa) tiene dos componentes; uno, podríamos decir, es un componente descriptivo; al otro Husserl lo denomina "un $X$ ".

\footnotetext{
${ }^{3}$ La razón que Husserl da al respecto es que "la percepción es un acto que determina pero no contiene significado" (LI, VI, § 5). Contra Smith y McIntyre pienso que Husserl señala aquí el camino correcto: la relación entre contenido mental demostrativo y contenido semántico no es directa.

${ }^{4}$ Según la corriente interpretativa iniciada por Føllesdal (1969) y desarrollada por él mismo y por autores como Dreyfus, McIntyre, Smith y Miller ( $c f r$. Smith y McIntyre 1982, y los ensayos de Dreyfus y Miller incluidos en Dreyfus 1982).
} 
Con esta denominación, Husserl pretende aludir al componente en el noema que corresponde al carácter singular de la manera en que se capta el objeto. Está claro que este rasgo de un noema, aunque sirve para diferenciar un noema de un contenido puramente descriptivo, no caracteriza completamente el contenido demostrativo de un estado perceptual, ya que ese rasgo se encuentra también en otros tipos de noemata. ${ }^{5}$

A esta fuente de incompletud en la teoría husserliana de la percepción hay que sumar las derivadas de otros aspectos distintivos de la misma. Así, Husserl sostiene que en la percepción intervienen decisivamente las sensaciones. Ahora bien, Husserl rechaza una concepción intencional o representacional de las sensaciones y la idea de una conexión inferencial entre sensaciones y percepción de objetos y propiedades, rasgo este último que diferencia su concepción decisivamente de la posición empirista. En efecto, según Husserl, el paso de la sensación a la percepción no es de naturaleza inferencial, sino, según parece, interpretativa: la percepción de un objeto o de sus propiedades sensibles requiere la "apercepción" (Auffassung), caracterizada por un sentido (Auffassungssinn). En la versión de Ideas, este sentido lo proporciona precisamente el noema del acto, o mejor, se identifica con este noema. Ahora bien, un noema es un tipo de entidad abstracta y por lo tanto atemporal, de modo que no forma parte de la corriente de conciencia. Pero en un acto específico de percepción (un fragmento de esa corriente), al noema perceptual de que se trate le corresponde una fase noética del acto. Así, expresado en los términos de la teoría de Ideas, un acto de percepción, y, en particular, un acto de percepción de un objeto singular, tiene dos fases, una puramente sensorial, ${ }^{6}$ que para Husserl no es intencional en absoluto, y otra noética, en la cual el noema correspondiente da cuenta del carácter intencional específico del acto.

En adelante vamos a servirnos del planteamiento husserliano para nuestros propósitos. A tal fin hay que observar que el término "fase" que aparece en los términos "fase sensorial" y "fase noética" sugiere

${ }^{5}$ Esta deficiencia ha sido reconocida entre los intérpretes de Husserl. Véase Smith y McIntyre 1982 (cap. IV, § 3.4, y en especial la p. 218).

${ }^{6}$ En las Investigaciones lógicas, Husserl habla del papel que en la percepción desempeñan las sensaciones (Empfindungen) ( $c f r$., por ejemplo, entre otros muchos pasajes, vol. II, parte I, pp. 75-76, 128, 129, 194-195, 197 de la edición original cuya paginación se reproduce en la edición crítica de la serie Husserliana). En Ideas introduce el término griego "hyle" (literalmente materia, pero más conspicuamente traducido como material sensorial) y habla de una "fase hylética", que algunos autores denominan también "fase sensorial". 
un orden de sucesión temporal: cuando acaba la primeramente nombrada, comienza la segunda. Sin embargo, probablemente Husserl no pretendía sostener que las fases fueran sucesivas y, en todo caso, al utilizar en adelante esa terminología husserliana, no es mi intención presuponer o postular tal estructura temporal, ya que parece razonable pensar que las dos "fases" se solapan temporalmente. Se establece así de un modo natural un marco en el que se desprenden del problema general de la relación entre sensación y percepción los tres problemas más concretos siguientes:

1. ¿Cómo hemos de caracterizar el componente distintivamente demostrativo del contenido de un estado perceptual?

2. ¿Cómo se ha de caracterizar la "fase sensorial" de un acto de percepción?

3. ¿Cómo caracterizar exactamente la relación entre la "fase sensorial" de tales estados y el contenido demostrativo (en términos husserlianos: entre la fase sensorial y la fase noética, atendiendo al carácter demostrativo de esos estados)?

En lo que sigue abordaremos estos tres problemas, en el orden en que se han formulado. Como veremos, el objetivo principal de este trabajo es presentar una solución parcial del tercero de ellos tomando como base propuestas concretas de solución a los dos primeros.

\section{Modos de presentación demostrativo-perceptuales de objetos}

Si caracterizamos de modo amplio el enfoque husserliano acerca de la percepción con la tesis de que hay un componente del contenido intencional que debe dar cuenta del "carácter demostrativo" preteoréticamente o preanalíticamente reconocido en ella, destacan en especial dos teorías husserlianas en este amplio sentido: la de John Searle y la de David Woodruff Smith. Las dos teorías tienen en común el que introducen en el contenido mismo un elemento contextual, y en ambos casos este elemento consiste en la relación causal del objeto percibido con el perceptor (o tiene mucho que ver con ella). También en ambos casos interviene de algún modo el carácter sensorial del verse causalmente afectado por el objeto.

Según la teoría de Smith — desarrollada explícitamente en relación con la perspectiva husserliana-, el modo de presentación del objeto es: "como ante el perceptor, y afectándolo sensorialmente, en la ocasión de percepción" (Smith 1984, p. 73). Debe entenderse que esta 
condición compleja forma parte del contenido del estado perceptual, es decir, "una parte del contenido fenomenológico de la percepción prescribe un objeto ante el perceptor afectándolo sensorialmente en la ocasión de percepción" (p. 73).

La cuestión, formulada en esos mismos términos, es exactamente cómo esa parte del contenido prescribe el objeto como estando ante el perceptor y afectándolo causalmente. Expresar la condición de manera cuantificacional es compatible con la caracterización que se acaba de dar: "Hay uno y sólo un objeto ante el perceptor afectándolo sensorialmente." El problema es que, por supuesto, esta formulación sería completamente inadecuada para caracterizar un componente demostrativo. Smith atiende implícitamente al problema, rechazando una interpretación descriptiva de su condición, pero creo que no hay nada en su caracterización positiva del modo de presentación demostrativo que efectivamente elimine esa posibilidad, con lo que no puede afirmarse que ha determinado suficientemente el carácter singular del contenido demostrativo ( $c f r$. Smith 1984, pp. 77 y 79).

El problema descrito se soluciona en principio en la propuesta de Searle al estar asegurado el carácter singular del contenido en cuestión por el hecho de que, en la condición de satisfacción del contenido del estado perceptual, se alude al episodio causal entre el objeto y el perceptor de una manera muy particular. La condición puede enunciarse aproximadamente así: "Hay un objeto y sólo uno ante mí [...] y tal objeto causa esta experiencia" (cfr. Searle 1983, pp. 48-49). La especie de "referencia demostrativa" — digámoslo así - que se hace a la propia experiencia perceptual cuyo contenido se está dando al especificar ese contenido hace que, prima facie, éste tenga cierto carácter singular a pesar de la forma cuantificacional general en que se da. ${ }^{7}$

Del modo indicado, según el análisis de Searle, a los estados o experiencias perceptuales los caracteriza una suerte de reflexividad. Esto ha sido reconocido como un elemento en principio positivo de la propuesta de Searle, pero se ha señalado al mismo tiempo la falta de análisis de esa especie de referencia que se hace a la propia experiencia; la propuesta de Searle estaría así infradesarrollada ( $c f r$. McDowell 1991, p. 217; García-Carpintero 1999, pp. 37-38).

Mi propia propuesta sigue la dirección señalada por Husserl por una senda distinta de la de Smith o Searle, a saber, desarrollando la

${ }^{7}$ La cuestión se examina detenidamente en las dos primeras secciones de mi artículo Fernández Prat 2006. 
idea de que la identificación demostrativa de un objeto está vinculada con la atención al mismo.

La idea de que la atención al objeto es un elemento crucialmente vinculado al carácter demostrativo de la percepción se debe a autores como Campbell, Brewer y Eilan (cfr. Campbell 1997, 1998 y 2002, Eilan 1998 y 2001, Brewer 1999). En la última sección presentaré breve y críticamente la posición de Campbell, el único en que puede encontrarse una propuesta específica acerca de la relación entre sensación y percepción, en particular con respecto a los tres problemas formulados al final de la primera sección.

Mi propuesta se basa en los siguientes principios:

(i) La atención a un objeto en la percepción es episódica: hay episodios, delimitados temporalmente, de atención.

(ii) El sujeto de la percepción se apercibe del episodio de atención en el que se encuentra.

(iii) Los estados perceptuales que tienen contenido demostrativo son estados en los que el sujeto atiende (perceptualmente) al objeto.

(iv) El elemento contextual que debe incluirse en la caracterización del contenido demostrativo de la percepción es, precisamente, el propio episodio de atención involucrado.

Creo que (i) es obviamente verdadero y que su verdad debe ser así reconocida por cualquier análisis de la noción de atención a un objeto. $^{8}$ El principio (iii) es la idea que tomo de las reflexiones de Campbell y Eilan. El principio (iv) constituye mi propia versión de la idea general de Smith y Searle de que es preciso incluir un elemento contextual en el propio contenido del estado perceptual, por decirlo así. El elemento contextual no es, como en sus propuestas, la relación causal con el objeto, sino el episodio de atención al mismo. Por último, el principio (ii) es otro elemento original de mi propuesta.

De acuerdo con estos principios, mi propuesta es que el contenido demostrativo de un estado perceptual consiste esencialmente en que el modo en que el objeto se presenta en ese estado queda adecuadamente especificado mediante la siguiente condición:

\footnotetext{
${ }^{8}$ Véase un análisis completamente adecuado para los propósitos aquí planteados en Peacocke 1998. Peacocke contrasta ahí la atención a un objeto con el tener la atención ocupada. Es la primera de estas dos nociones la que se utiliza en mi propuesta.
} 
(MPD) (ser) atendido en este episodio de atención.

En principio, esta condición puede considerarse como razonablemente individuadora (el "elemento singular") y también como epistémicamente accesible — de algún modo - al sujeto (como debe serlo todo modo de presentación). La propuesta, claro está, necesita desarrollo y ulterior justificación (p. ej., el último punto mencionado, es decir, el principio (ii)), pero me siento exonerada de presentar aquí este desarrollo, pues razones de extensión aconsejan remitir a mi presentación en otro lugar (cfr. Fernández Prat 2006). ${ }^{9}$

\section{El problema de cómo caracterizar la "fase sensorial"}

La propuesta que voy a hacer para concretar la "fase sensorial" es una versión de la teoría de los datos sensoriales de la percepción. Está muy claro, sin embargo, que cualquier propuesta de este tipo parece enfrentarse a dificultades formidables o incluso, según muchos, insuperables, de manera que explicaré por qué es razonable pensar que la versión de la teoría que adopto supera esas dificultades. ${ }^{10}$

En las teorías tradicionales de los datos sensoriales se sostiene que la conciencia de éstos constituye el fundamento del conocimiento empírico, lo que choca frontalmente con el hecho de que las formas clásicas de fundamentismo epistemológico son rechazadas de manera prácticamente unánime en la filosofía actual. También el internismo

${ }^{9}$ En ese artículo se sostiene que el carácter singular del modo de presentación demostrativo se refleja en una interpretación referencial de una descripción basada en la condición (MPD), lo que dio lugar a la objeción expresada en Grimaltos y Moya 1996 de que un estado perceptual alucinatorio carecería entonces de contenido. Este problema no se plantea al reconocer dos tipos de contenido, como se hace en el presente artículo. Sin embargo, en todo caso creo ahora que el carácter singular del modo de presentación demostrativo y la inmediatez del objeto percibido (cuando lo hay) quedan suficientemente reconocidos en una interpretación atributiva de la descripción que incluye una referencia demostrativa al episodio de atención, la cual, por supuesto, ha de ser explicada independientemente, como se hace en el artículo mencionado, pp. 140 y ss.

${ }^{10}$ Las dificultades comienzan en el propio Husserl, pues éste niega que la fase sensorial sea intencional, lo que significa - dado el vínculo que para él tienen intencionalidad y conciencia - que el perceptor no tiene, estrictamente, ningún tipo de conciencia de esa fase. Ésta es la principal razón por la que la mayoría de los intérpretes de Husserl rechazan el uso ocasional que hace el propio Husserl de la terminología de los "datos sensoriales", ya que este término de la tradición empirista se suele asociar con la idea central de esa tradición, a saber, que es precisamente de tales datos de lo que se tiene conciencia primariamente en la percepción. Así, los datos sensoriales constituyen en esa tradición lo dado inmediatamente a la conciencia, y, como tal, el fundamento último del conocimiento empírico. 
acerca de lo mental con el que se suele asociar a las teorías de los datos sensoriales las pone en una mala situación en un contexto filosófico como el actual, dominado en buena parte por diversas formas de externismo acerca del contenido mental, a partir de los trabajos clásicos de Kripke y Putnam en filosofía del lenguaje, y de Burge en filosofía de la mente.

Según un acuerdo prácticamente unánime en la actualidad, un argumento a favor de una teoría filosófica de la percepción que postule datos sensoriales no puede ser directo; en especial no puede basarse en el llamado principio fenoménico ("si sensorialmente a un sujeto le parece que hay algo que posee una determinada propiedad sensorial, entonces hay algo de lo que se percata el sujeto que posee esa propiedad sensorial"). En todo caso, el argumento habría de recurrir a la mejor explicación; es decir, habría de explicarse por qué la teoría de los datos sensoriales constituye en definitiva - cuando todos los elementos se tienen en cuenta- la mejor explicación filosófica disponible de la percepción, lo que, en el clima filosófico actual, hace urgente indicar cómo puede esa doctrina escapar a las objeciones al fundamentismo epistemológico empirista y a cualquier forma rechazable de internismo con respecto al contenido de los estados mentales.

Librarse de lo primero no es algo implausible en el contexto de la discusión epistemológica actual. En efecto, una posición de tipo dogmatista (cfr. Pryor 2000), defendida argumentativamente como una opción mejor que las restantes para replicar a los argumentos escépticos sobre el conocimiento del mundo externo, sostiene — para el caso aquí pertinente- que algunos juicios perceptuales están legitimados por el mero hecho de que el sujeto tiene determinadas sensaciones, pues dichas sensaciones son constitutivas de alguno de los conceptos que conforman los juicios en cuestión; es decir, por ejemplo, quien es capaz de hacer el juicio "Esto es rojo" posee el concepto de rojo y poseer dicho concepto requiere precisamente hacer, en condiciones normales, dicho juicio al experimentar cierto tipo de sensación cromática (cfr. Peacocke 1992, p. 7). Esta posición muestra así cierta afinidad con el fundamentismo empirista y se enfrenta a posiciones epistémicas internistas que requieren que lo que hace epistémicamente legítimo un juicio perceptual ha de estar "en el espacio de las razones" (McDowell 1994). Sin embargo, y contra el fundamentismo empirista tradicional, se sostiene que en un proceso de búsqueda de equilibrio reflexivo cualquier juicio que resulte legitimado del modo descrito puede ser revisado y eventualmente resultar revocado ( $c f r$. García-Carpintero 2005 para una sucinta exposición y defensa de la posición aquí descrita). 
Podría parecer más difícil librarse de cualquier forma rechazable de internismo pues las teorías de los datos sensoriales son teorías que defienden que aquello de lo que somos inmediatamente conscientes en la percepción tiene un carácter subjetivo, y parece ser exactamente esto lo que caracteriza a una posición internista. Sin embargo, la versión de la teoría de los datos sensoriales que aquí se adopta no es internista, como vamos a ver brevemente.

La teoría de los datos sensoriales, en la versión que se acepta aquí, sostiene que los rasgos fenoménicos de la experiencia tienen ontológicamente un carácter doble: cada rasgo fenoménico corresponde a dos propiedades, subjetiva o dependiente de la mente la una, material la otra, entendiendo por propiedad dependiente de la mente una que no podría existir sin que sujeto alguno se apercibiera de ella, y por entidad material una cuya existencia no depende de ese apercibimiento. $^{11}$

Una teoría así podría rechazarse de buen comienzo si la fenomenología de la percepción fuera claramente incompatible con ella. Ahora bien, el rasgo más importante de esa fenomenología parecer hallarse en el carácter diáfano de la percepción: su capacidad para, en los casos normales, ponernos en contacto inmediato con objetos y propiedades materiales de los mismos y ese rasgo no comporta necesariamente una alternativa excluyente, es decir, no es incompatible con una teoría que afirma que en casos de percepción tenemos, respecto de un rasgo fenoménico, conciencia tanto de la ejemplificación de una propiedad subjetiva como de la de una propiedad material, sólo que normalmente nuestra atención está puesta sobre todo en la segunda. Una analogía puede ayudar aquí. Cuando oímos hablar a otra persona y la entendemos, ¿de qué somos conscientes de forma inmediata, de las cosas (acaecimientos, etc.) que explica o de las palabras que utiliza? De las dos cosas, en diversos grados según el grado de atención que prestemos a una cosa o la otra en las diversas situaciones. Normalmente prestamos mayor atención a lo que se dice o explica, como se revela en el hecho de que recordamos esto mejor que las palabras concretas que se utilizaron en la explicación, pero lo que se explica con las palabras sólo nos es accesible si las palabras lo son. Lo que se dice o explica se presenta entonces, podemos decir, directamente a nuestra conciencia (la conciencia auxiliar de las palabras no nos impediría otorgar este carácter directo a la relación). Pues bien, este hecho, que Husserl fue el primero en señalar, fue aducido por C.D. Broad - uno de los más destacados defensores de la teoría

\footnotetext{
${ }^{11}$ Por lo que sé, esta posibilidad se apunta por primera vez en Perkins 1983.
} 
de los datos sensoriales - como ejemplo de relación no inferencial ni tampoco meramente causal, precisamente para iluminar por analogía la relación entre datos sensoriales y objetos o propiedades materiales.

Vemos pues que al menos algunos teóricos de los datos sensoriales sugirieron que la conciencia no inferencial de un objeto (una conciencia, en este sentido, inmediata), una propiedad o un hecho material puede ser compatible con la conciencia de un dato sensorial. Es cierto que se puede ser escéptico sobre el valor último de la analogía aducida, pero es difícil no admitir que ésta motiva la duda de si la diafanidad de la experiencia perceptiva es en realidad incompatible con cierto carácter irreduciblemente subjetivo de esa experiencia. Parece pues prudente pensar que lo que un rasgo fenoménico sea, ontológicamente hablando, no tiene por qué desprenderse meramente ni de su carácter general como tal rasgo fenoménico ni del carácter específico que tenga. Dicho de manera sucinta: la ontología de los rasgos fenoménicos no se desprende de su fenomenología. Este principio se respeta en la discusión contemporánea, también entre quienes insisten en el carácter diáfano de la percepción, pues si se concluye que aquello de lo que se es inmediatamente consciente son (únicamente) objetos o propiedades materiales se aducen razones ulteriores para ello. Esto vale tanto para la posición disyuntivista defendida en Snowdon 1981 y McDowell 1982, como para la intencionalista de Harman 1989, Tye 1995 o Dretske 1995.

La teoría del "doble aspecto" de los rasgos fenoménicos hace posible sostener que en un caso concreto de experiencia perceptual siempre se ejemplifican propiedades subjetivas (al menos una propiedad subjetiva) y que en los casos no alucinatorios se ejemplifican también propiedades materiales. En esto último, la teoría coincide con el intencionalismo - un rasgo de las apariencias ejemplifica en condiciones normales una propiedad material - y puede aceptar además la versión funcional-teleológica (defendida con fuerza en Dretske 1995), sosteniendo que la función de una experiencia perceptual es justamente reconocer o detectar una propiedad material, con lo que se establece una dependencia de las propiedades subjetivas respecto de las materiales que habremos aún de puntualizar. Según la teoría, pues, un rasgo fenoménico tiene carácter representacional. Al mismo tiempo, dado que, según la teoría, un rasgo fenoménico no se agota en su carácter representacional, la teoría coincide con la posición fenomenista defendida en Block 1995 y Chalmers 1996 (y con las versiones cartesianas de la teoría de los datos sensoriales) en que los rasgos de las apariencias son subjetivos, es decir, una experiencia perceptual se individualiza por las propiedades subjetivas que en 
ella se ejemplifican. Con ello la teoría reúne lo que se ha aducido como ventajas de una y otra posición. En particular, al no separar lo fenoménico de lo representacional, la teoría logra superar las serias objeciones que se presentan en Dennett 1988 y Akins 1993 contra esa separación, objeciones que, en mi opinión, no han encontrado respuesta adecuada por parte de los defensores de una concepción fenomenista de los rasgos fenoménicos.

No es posible desarrollar aquí esta argumentación. En todo caso, mi propósito en este trabajo no es defender la versión mencionada de la teoría de los datos sensoriales, ${ }^{12}$ sino utilizarla para clarificar algunos aspectos cruciales de la relación entre sensación y percepción, para lo cual bastará esbozar sus rasgos más relevantes en el presente contexto.

Ante todo, ¿se trata de una teoría externista o internista? Una teoría internista acerca de los datos sensoriales sostendría que cualquiera de estos datos puede ser individualizado con entidades subjetivas o dependientes de la mente. ¿Sucede esto según la teoría en cuestión? Para responder a la pregunta es necesario distinguir entre un dato sensorial como tipo y la ejemplificación del mismo. Como se ha adelantado ya, la teoría sostiene que, en efecto, cada ejemplificación particular de un dato sensorial puede individualizarse exclusivamente mediante entidades (propiedades) subjetivas (es lo que sucede en casos de alucinaciones). Y, sin embargo, el dato sensorial considerado como tipo requiere propiedades materiales para su individuación, en el sentido de que una condición necesaria para la existencia del tipo es que el sujeto esté o haya estado en relación con tales propiedades; de modo que no es posible que todas las ejemplificaciones de un dato sensorial puedan individualizarse mediante entidades subjetivas. En este sentido, la teoría se caracteriza por un externismo modesto.

De manera sucinta, los rasgos de la teoría necesarios para los presentes propósitos son los siguientes. La teoría afirma que en todo estado perceptual (incluyendo estados en los que el sujeto está percibiendo realmente un objeto material $O$, y estados en los que alucina

\footnotetext{
${ }^{12}$ Naturalmente, presupongo que la teoría en cuestión es defendible. Parte de los argumentos importantes para una teoría de la percepción de los datos sensoriales se encuentra en Jackson 1977 y Perkins 1983. En García-Carpintero 2001 se presenta, en forma sistematizada, la versión concreta de la teoría a la que me adhiero y se delinea un argumento muy atendible que defiende que la teoría de los datos sensoriales, en esa versión concreta, suministra el mejor análisis filosófico disponible de la percepción. En cualquier caso, en García-Carpintero 2002 se argumenta - convincentemente, en mi opinión - que esa versión constituye una alternativa razonable a las teorías disyuntivistas (radicales), intencionalistas y fenomenistas.
} 
$O$ ) está involucrada una experiencia $E$, la cual consiste en una relación de apercibimiento (awareness) entre el sujeto y un objeto del tipo de los eventos, $s-d(E)$ (el dato sensorial de $E$ ), el cual a su vez consiste en la ejemplificación de un complejo de propiedades fenoménicas dispuestas de forma espacio-temporalmente estructurada desde un punto de vista egocéntrico ( $c f r$. García-Carpintero 2001, p. 37). Acerca de estas propiedades fenoménicas - los qualia en el sentido ontológicamente subjetivo-, la teoría hace tres afirmaciones que son de interés en el presente contexto: i) no son reducibles a propiedades de los objetos materiales percibidos (concretamente a las propiedades materiales observables en los mismos); ii) ocupan puntos en espacios que son isomorfos a espacios de propiedades materiales observables, ${ }^{13}$ iii) la ejemplificación de cada propiedad subjetiva implica la ejemplificación de una propiedad material observable homóloga en su espacio correspondiente (o al menos de una propiedad similar a ella, es decir, cercana en el espacio cualitativo correspondiente) en algún caso posible de percepción; no necesariamente en el mismo caso en el que la propiedad fenoménica subjetiva se ejemplifica (en el sentido de iii, la dependencia de las propiedades subjetivas respecto de las materiales en la percepción se concreta como una dependencia funcional); y iv) debido a su eficacia causal, las propiedades fenoménicas subjetivas (como también las propiedades observables) deben poseer alguna naturaleza categorial específica, además de la que les corresponde por el hecho de ocupar sus lugares respectivos en el espacio cualitativo (García-Carpintero 2001, p. 29).

Hasta aquí la parte del análisis explicativo de la percepción que concierne estrictamente a los datos sensoriales, en los que, según la presente propuesta, consiste la "fase sensorial" del estado de percepción. Ésa es la parte que realmente nos atañe. En el contexto del tema que nos ocupa en este trabajo, recurrir a una teoría de los datos sensoriales bien articulada — en caso de que ésta sea filosóficamente viable, claro está - presenta la ventaja de superar las vaguedades que ofrecen teorías como las de Searle y Smith, consideradas brevemente en la sección anterior, las cuales recurren respectivamente a "expe-

\footnotetext{
${ }^{13}$ Se rechaza así que predicados como "es rojo" se apliquen en sentido unívoco a cosas materiales y a datos sensoriales (como se sostiene en Jackson 1977, pp. 74-81 y 103). Como se ha avanzado, la teoría se basa en la idea de Perkins de los dos aspectos — sensorio (sensous) y material o físico - de los rasgos fenoménicos ( $c f r$. Perkins 1983, especialmente el capítulo 8), aplicada de forma que se recoge la idea de que los parecidos en los rasgos fenoménicos se dan según diferentes dimensiones, rasgo este último que se defiende en obras clásicas como Goodman 1978 y se subraya en Shoemaker 1975 y especialmente en Clark 1993.
} 
riencias" ( $c f r$. Searle 1983, p. 46) y al ser "afectado sensorialmente" (sensously affected), sin explicar en qué consisten tales experiencias o ese verse sensorialmente afectado.

\section{Datos sensoriales y modos de presentación demostrativo-perceptuales}

Pasamos finalmente a abordar el problema cuya solución —al menos parcial - constituye el objetivo central de este trabajo, el problema de la relación entre la "fase sensorial" y la "fase noética" representada por el contenido demostrativo de los estados perceptuales de los que aquí se trata.

Antes de entrar de lleno en el problema, es relevante recordar que los filósofos que analizan la percepción están divididos en cuanto a si la percepción de objetos es más fundamental que la percepción de estados de cosas (percepción con contenido proposicional), o bien ocurre a la inversa, o bien, por último, ambas son igualmente fundamentales. Por fortuna, aquí no necesitaremos dilucidar esta cuestión, pues la propuesta que se planteará es neutra con respecto a las tres posibilidades. En el enfoque que hemos adoptado, los objetos se presentan demostrativamente (sección 2), tanto si constituyen el contenido de un estado de percepción, como si forman parte de una proposición que constituye propiamente el contenido del estado.

Dicho esto, en aras de una mayor concreción, vamos a considerar los modos demostrativo-perceptuales de presentación como parte de los contenidos de un juicio de percepción. Así, aceptamos que un estado perceptual del tipo considerado, cuando es exitoso, es decir, cuando verdaderamente se trata de un estado de percepción, nos pone en una relación inmediata (no inferencial) con un objeto material a la que calificamos de "demostrativa", precisamente por su inmediatez. Hemos precisado esta intuición en términos de modos de presentación demostrativo-perceptuales y hemos explicado sucintamente en qué consisten éstos. Ahora añadimos que consideramos estos modos de presentación como parte constituyente de juicios perceptuales a los que, precisamente por contener tales modos de presentación, denominamos juicios perceptuales demostrativos.

Ahora bien, por otro lado aceptamos que en todo estado perceptual, incluidos, claro está, los estados a los que acabamos de aludir, está involucrada una experiencia que consiste en que un sujeto se apercibe o percata de un dato sensorial. De modo que en los estados perceptuales que nos interesan tenemos, por una parte, los datos 
sensoriales, y, por la otra, los modos demostrativo-perceptuales y los juicios perceptuales de los que forman parte.

Mi primera sugerencia es que resulta adecuado conceptualizar esta situación utilizando la noción de contenido. Tenemos entonces dos tipos de contenido: el contenido consistente en el modo de presentación y el juicio que contiene ese modo de presentación, y el contenido consistente en el dato sensorial. Se trata, según creo, de tipos fundamentalmente diferentes de contenido.

Como se ha podido ver, acepto aquí que los modos de presentación demostrativo-perceptuales forman parte del contenido conceptual, con lo que sigo una tradición reciente pero poderosa. ${ }^{14}$ En relación con los datos sensoriales, es difícil poner en duda que constituyen un tipo de contenido no conceptual, si es que a ellos les es aplicable la noción de contenido, cuestión que vamos a examinar brevemente.

La noción más clara de contenido exige que algo que tiene un determinado contenido incluya ciertos estados de cosas y excluya otros. En otras palabras, la noción de contenido se vincula indisolublemente con la de contenido representacional. Según esto, si nos atuviéramos a una noción de dato sensorial que divorciara totalmente los datos sensoriales de los aspectos representacionales, sería inadecuado decir que los datos sensoriales constituyen un tipo de contenido. Pero la versión de la teoría de los datos sensoriales a la que nos adherimos aquí rechaza decididamente ese divorcio. De modo que el obstáculo desaparece.

Una vez que está despejado el camino para considerar los datos sensoriales como contenido de los estados perceptuales, hay pocas dudas de que se trata de un contenido no conceptual. Todas las versiones, tradicionales o no, de las teorías de datos sensoriales los contrastan con los juicios y con los conceptos que constituyen a éstos. La razón de fondo es que los datos sensoriales no pueden proporcionar razones (aunque puedan proporcionar otro tipo de legitimación, como mencionamos en la sección anterior), mientras que los conceptos son precisamente lo que constituye los (contenidos de los) juicios, que son la clase de entidad mediante la cual se ofrecen razones.

Admitido que en un estado perceptual del tipo que nos ocupa pueden discernirse dos tipos de contenido, su contenido no conceptual - el dato sensorial del que el sujeto se percata - y su contenido conceptual — contenido demostrativo-, hemos de reconocer enseguida

${ }^{14}$ La tradición de considerar los modos de presentación demostrativos y los juicios correspondientes como contenido conceptual procede de Evans 1982. McDowell 1994 contribuyó decisivamente a darle la fuerza que ha adquirido en tiempos más recientes. 
que estos dos contenidos no van, por decirlo así, cada uno por su cuenta. De alguna manera, el contenido del juicio que involucra un modo de presentación demostrativo debe corresponder al dato sensorial. El problema de la relación entre sensación y percepción se nos presenta así parcialmente como el problema de determinar esta correspondencia entre los dos tipos de contenido. ${ }^{15}$

Es, pues, la noción de contenido correspondiente, limitada al caso de un modo de presentación demostrativo y un dato sensorial (o componentes del mismo), la que me propongo explicar aquí. Lo que presentaré en un momento es una condición necesaria de que un modo de presentación demostrativo perceptual corresponda a un dato sensorial (o, más bien, a componentes del mismo); dicho de otro modo, una condición necesaria de que un juicio perceptual que contenga un modo de presentación demostrativo-perceptual corresponda parcialmente a un dato sensorial. Creo que esta condición es la parte más importante, y también la más difícil, en la formulación de una condición necesaria y suficiente. Considero que la parte restante es relativamente sencilla, aunque no proseguiré aquí esta cuestión.

El problema que hemos de tratar se presenta como algo que ofrece cierta dificultad. Notemos ante todo que, incluso si estamos dispuestos a admitir que los datos sensoriales son de algún modo constitutivos de los modos de presentación en juicios perceptuales, sería erróneo tomar los datos sensoriales mismos como modos de presentación demostrativos. Claramente, sería insuficiente apuntar que un dato sensorial particular debe proporcionar los ingredientes - por decirlo así - del juicio perceptual completo con el que está asociado. Tampoco sería una solución identificar el modo de presentación demostrativo-perceptual simplemente con una especie de "conglomerado" de los qualia que se ejemplifican en el dato sensorial, puesto que, en ese caso, el modo de presentación sería algo puramente cualitativo. Dos experiencias de objetos respectivamente diferentes,

\footnotetext{
${ }^{15}$ García-Carpintero, cuya versión de la teoría de los datos sensoriales hemos adoptado, es consciente de que deja este problema abierto. En García-Carpintero 2001, tras afirmar que, de acuerdo con esa teoría de los datos sensoriales, una experiencia perceptual causa - en condiciones normales - un juicio perceptual con un contenido correspondiente, añade lo siguiente: "Al hablar de un contenido "correspondiente', paso por alto los difíciles asuntos que tienen que ver con el [...] hecho de que el contenido de las experiencias perceptuales es no conceptual, mientras que el contenido de los juicios que en ellas se basan es contenido conceptualizado" (García-Carpintero 2001, nota 34). Hay que señalar que García-Carpintero acepta que los juicios perceptuales pueden tener contenidos singulares ( $c f r$. García-Carpintero 2002, p. 209).
} 
aunque idénticos en lo cualitativo, estarían entonces asociadas al mismo juicio perceptual, de manera que la posibilidad de que el propio objeto percibido - causante de dichas experiencias - contribuya de algún modo (aunque sea indirectamente) a la individuación del contenido intencional del estado perceptual estaría descartada de antemano, algo que parece ir en contra del espíritu de todo el enfoque que estamos prosiguiendo con el énfasis en el "elemento demostrativo" de la percepción y la insistencia en que ese objeto está "directamente presente."

Mi explicación de la correspondencia buscada recurre, aquí también, a la noción de atención. Como se verá, el elemento intuitivo de esta noción que es relevante en el presente contexto es que la atención perceptual a un objeto implica "seleccionarlo" o "entresacarlo" de su trasfondo perceptivo, con el propósito general de obtener alguna información del entorno o una información mejor.

Parece plausible identificar el trasfondo del cual se "entresaca" o "selecciona" de algún modo un objeto en un episodio de atención a éste con los rasgos con que se nos presenta al percibirlo. Mi propuesta es que cuando "seleccionamos" o "entresacamos" un objeto de su trasfondo perceptual, lo hacemos en virtud de "ligar" esos rasgos fenoménicos. Afirmar esto exige cierta concepción realista y subjetivista — al menos parcialmente subjetivista- de estos rasgos, es decir, el tipo de concepción que de los mismos se plantea en la versión de la teoría de los datos sensoriales que hemos adoptado.

En el marco teórico que asigna un papel central a los datos sensoriales puede aceptarse que los diversos rasgos se ejemplifican independientemente. ${ }^{16}$ Pero la ejemplificación de un rasgo ocurre en el contexto del tipo de percepción que estamos analizando - la

\footnotetext{
${ }^{16}$ Aquí se plantea la cuestión de si puede hablarse de la identificación de qualia específicos en nuestra conciencia. Creo que los procedimientos de identificación de qualia que se elaboran en Clark 1993 parecen inclinarnos a sostener que, efectivamente, se da esa identificación. Además, el tipo de tarea sistemática de que se habla en el texto parece requerir la admisión de qualia individuales. Sin embargo, no considero definitivo el tipo de atomismo que se sugiere en el texto. Pienso que además de reconocerse que los qualia perceptuales se presentan como parte de acaecimientos sensoriales complejos (los datos sensoriales), debería admitirse que también pueden formar parte de otras propiedades fenoménicas complejas, y quizá éstas no siempre se puedan descomponer en elementos constitutivos, debido a efectos tipo Gestalt. También es preciso considerar seriamente la posibilidad de propiedades fenoménicas relacionales. Otra limitación importante de mi análisis es la de no tener en cuenta los aspectos dinámicos de la percepción que, como han argumentado Evans y Campbell, son muy relevantes para el pensamiento demostrativo y que algunos consideran esenciales para la percepción de objetos ( $c f r$. Mulligan 1995, p. 197).
} 
"percepción demostrativa" - cuando se está atendiendo a un objeto (material). En ese caso no ocurre que simplemente se ejemplifiquen - simultánea o sucesivamente - diversos rasgos (y, por lo tanto, según la teoría, subjetivamente una serie de qualia), sino que parece darse además algún tipo de operación "combinadora" o "sintetizadora" de la mente. Podemos decir — apropiándonos de un término utilizado por teóricos de los datos sensoriales como Moore y Jacksonque al sujeto se le presentan los rasgos fenoménicos del objeto como pertenecientes al objeto, lo que requiere lo que denominamos el $l i$ gado de tales rasgos. Mi caracterización de esta noción es la siguiente (siendo $Q_{F}, Q_{F^{\prime}}$, etc., los qualia que se ejemplifican en un estado perceptual que presenta los rasgos fenoménicos $F, F^{\prime}$, etc.):

Un rasgo fenoménico $F$ pertenece a un objeto material $M$ en un estado perceptual (demostrativo) de un sujeto $S$ en el que éste atiende a $M$ si y sólo si

(i) un acaecimiento que involucra $M$ causa que $Q_{F}$ se ejemplifique en $S$.

(ii) hay otros rasgos fenoménicos $F^{\prime}, F^{\prime \prime} \ldots$ con respecto a los que se cumple lo siguiente: 1) $Q_{F^{\prime}}, Q_{F^{\prime \prime}}$ se ejemplifican en $S$; 2) esa ejemplificación es igualmente causada por $M$; y 3 ) en el estado perceptual de $S$ esos rasgos se ligan con $F$ (o alternativamente: $S$ los liga con $F){ }^{17}$

En la teoría de los datos sensoriales que adoptamos, ligar los rasgos fenoménicos tiene, por decirlo así, una doble dimensión. Se vinculan entre sí las ejemplificaciones de propiedades subjetivas o qualia - causadas por el objeto - correspondientes a propiedades materiales manifiestas del mismo, una correspondencia que viene dada por la significación externa de los qualia ( $c f r$. el rasgo iii de la explicación presentada al final de la sección anterior). Así, en los casos de percepción, ligar los rasgos fenoménicos corresponde con el hecho de que las propiedades manifiestas del objeto forman, por llamarlo así, un haz en él. Por ello, el ligado que se da al seleccionar o entresacar el objeto del trasfondo en el que se presenta tiene tanto una vertiente subjetiva - el vincular entre sí los qualia cuya ejemplificación es causada por

\footnotetext{
${ }^{17}$ Compárese con la formulación de Jackson 1977, p. 171. Aparte de las diferencias debidas a las distintas versiones subyacentes de la teoría de los datos sensoriales, la diferencia principal se da en la segunda condición de la definición, la cual utiliza la noción de ligado de rasgos fenoménicos.
} 
el mismo-, como una vertiente objetiva — el vincular entre sí las propiedades del objeto que ya están ligadas en él formando un haz de propiedades - ${ }^{18}$ En consecuencia, el apercibirse o percatarse de un dato sensorial causado por un objeto, que consiste en la ejemplificación de una serie de qualia, resulta, con la atención al mismo, en un apercibirse de las propiedades observables o manifiestas del objeto, podríamos decir, como formando una unidad.

Puede verse cuál es mi propuesta para resolver (parcialmente) el problema de la relación entre la "fase sensorial" de un estado perceptual y el modo de presentación demostrativo. Como ya vimos, la presentación demostrativa de un objeto se da al atender a ese objeto. A esto añadimos ahora que la atención al objeto es necesaria (y también suficiente, aunque no desarrollamos aquí este aspecto) para que tenga lugar la operación sintética que denominamos ligado de los rasgos fenoménicos. De ese modo, la atención resulta ser la bisagra que conecta los dos mundos, el sensorial y el propiamente perceptual. ${ }^{19}$

Es fácil precisar ahora una condición necesaria de la noción de correspondencia entre un dato sensorial y el juicio perceptual correspondiente a un estado perceptual de tipo demostrativo. Sea $J$ un juicio perceptual vinculado a un estado perceptual del tipo relevante, o constitutivo de él (que, para simplificar, supondremos exitoso). $J$ contiene así un modo de presentación demostrativo-perceptual, el cual, según la propuesta sobre estos modos de presentación formulada en la sección 2, involucra esencialmente un episodio de atención perceptual a un objeto (llamemos $A^{*}$ a este episodio). Sea $s$ - $d$ un dato sensorial consistente en la ejemplificación de los qualia $Q_{F^{\prime}}, Q_{F^{\prime \prime}}, \ldots$ Decimos que

(C) El dato sensorial $s$-d corresponde al juicio $J$ sólo si los rasgos $F^{\prime}, F^{\prime \prime}, \ldots$ pertenecen al objeto al que se atiende en $A^{*}$.

Sobre la base de la condición de correspondencia (C), la caracterización de otras nociones que perfilan más detalladamente el vínculo entre juicios perceptuales y datos sensoriales no ofrece dificultades.

\footnotetext{
${ }^{18}$ Dado que las propiedades manifiestas están ya "ligadas" en el objeto, la vertiente objetiva del ligado ha de considerarse propiamente como un reconocimiento de esas propiedades como ligadas o formando un haz.

${ }^{19}$ Nótese que, de acuerdo con la presente explicación, la relación causal no forma parte de aquello de lo que el sujeto se apercibe en el acto de percepción, algo que sería inadecuado requerir, según se mencionó en relación con las propuestas de Searle y Smith.
} 
Así, podemos decir que los modos de presentación constitutivos de los juicios perceptuales — siempre teniendo en cuenta juicios perceptuales del tipo mencionado anteriormente- están informados por los datos sensoriales correspondientes a tales juicios (nótese que aquí se apela implícitamente a los episodios de atención esenciales en la caracterización de la correspondencia). Los modos de presentación perceptuales (de objetos) pueden caracterizarse entonces como los que están informados por datos sensoriales.

Finalmente, podemos considerar que los modos de presentación están constituidos por rasgos fenoménicos en el sentido que la siguiente definición hace explícito:

Si 1) un sujeto $S$ se apercibe de un dato sensorial $s$ - $d$, lo que consiste en la ejemplificación de los qualia $\left.Q_{F^{\prime}}, Q_{F^{\prime \prime}}, \ldots, 2\right)$ el sujeto liga $F^{\prime}, F^{\prime \prime}, \ldots$ y 3) el modo de presentación $m$ está informado por $s$ - $d$, entonces $F^{\prime}, F^{\prime \prime}, \ldots$ constituyen $m$.

\section{Discusión: ¿un recurso a la ciencia cognitiva?}

El problema que, desde una perspectiva husserliana amplia, se planteó en la sección 1 sobre cómo caracterizar la relación entre las fases "sensorial" y "noética" o conceptual de la percepción tiene, como hemos visto, visos de solución apelando a las nociones de atención y ligado. Mi propuesta, sin embargo, no es la única que recurre a esas nociones. En Campbell 1997 y 2002 se recurre a ellas, por lo que a primera vista podría parecer que mi propuesta es muy cercana o coincidente con la de Campbell. En realidad, y a pesar de que la mía ha sido parcialmente inspirada por el estudio de la suya, las propuestas están filosóficamente muy alejadas, como paso a explicar brevemente.

Lo esencial para señalar las diferencias es concentrarnos en los términos de la relación. Para Campbell, uno de esos términos es lo que él llama "contenido imaginístico" (imagistic content) y el otro un modo de presentación que se obtiene por el ligado (binding) de los "ingredientes" o elementos constituyentes de ese contenido imaginístico. Sin embargo, tanto la noción de contenido imaginístico de que habla Campbell como la noción de ligado a la que recurre su explicación son nociones de teorías empíricas de la atención selectiva de la ciencia cognitiva (Campbell señala especialmente el trabajo sobre atención selectiva de Anne Treisman y colaboradores; en relación con la primera noción; cfr. entre otros, Treisman 1986, 1988 y Treisman y Gormican 1988.) Ciertamente, Campbell no hace de ningún 
modo una aplicación rutinaria de esas teorías, sino que hay diversos aspectos originales en su propuesta, como el énfasis en el papel que desempeñan los elementos a los que se aplica la operación de ligado, a los que se agrupa bajo una terminología sugerente ("contenido imaginístico").

En cualquier caso, el objetivo de Campbell es vincular directamente las nociones de una teoría filosófica del contenido mental con nociones y resultados de teorías empíricas. Concretamente, Campbell supone, de acuerdo con su interpretación de tales teorías, que la atención hace que mecanismos que operan en el sujeto seleccionen las porciones adecuadas del "contenido imaginístico" de la percepción proporcionando así la conciencia plena del objeto percibido. Su propuesta es entonces que las condiciones de identidad de los modos de presentación demostrativos - a los que Campbell se refiere frecuentemente como "sentidos (demostrativos)" o también como "demostrativos perceptuales" e incluso simplemente como "demostrativos"están determinadas por los procesos que se dan en los sujetos (al operar los mecanismos mencionados) cuando atienden perceptualmente a un objeto. A tales procesos se refiere Campbell como "métodos de selección" (de las porciones pertinentes de los contenidos imaginísticos), y postula específicamente que a diferente contenido imaginístico o diferente método de selección le corresponde un modo de presentación distinto, y que el mismo método de selección operando sobre el mismo contenido imaginístico determina el mismo modo de presentación (cfr. Campbell 1997, pp. 60-61; 2002, pp. 88-90).

La impresión inmediata frente a la propuesta de Campbell es que, por decirlo así, está completamente fuera de lugar, pues, por un lado, al recurrir a la noción de modo de presentación o sentido, parecería como si su asunto fuera especificar el contenido de la experiencia perceptual sirviéndose de nociones - en la tradición fregeana- que la describen en el nivel personal (dicho en la conocida terminología introducida por Dennett), mientras que los contenidos imaginísticos o los métodos de selección a los que recurre para especificar esos sentidos (o sus condiciones de identidad), si se dan, ocurren en el nivel subpersonal, pues se trata de entidades o procesos caracterizables completamente en términos de procesamiento de la información.

Campbell es, desde luego, plenamente consciente de que su posición implica un salto del nivel personal al nivel subpersonal. Pero en Campbell 1998 ofrece un interesante - aunque algo intrincadoargumento que mostraría que, en el caso presente y otros similares, tal salto es legítimo. 
Campbell se apoya específicamente en el caso de las conectivas. El modo de presentación o sentido de una conectiva está esencialmente vinculado a hallar y justificar las implicaciones de proposiciones que involucran esa conectiva. Quien capta, pues, el sentido de esa conectiva está captando, afirma Campbell, el objeto de procedimientos (a saber, los procedimientos utilizados al hallar y justificar tales implicaciones) de los cuales no tiene conciencia (es decir, está captando para qué son tales procedimientos). Si no se captara el sentido de la conectiva, se podrían seguir tales procedimientos, pero no se entendería su objeto y con ello no se tendría ninguna comprensión de por qué está justificada su intervención. Esto es lo que, según Campbell, apoya la tesis de que la noción de modo de presentación surge cuando tratamos de explicar la corrección de procedimientos determinados para extraer las consecuencias de ciertas proposiciones o (como en el caso de los modos de presentación demostrativos) para verificarlas.

Sobre esta base general, Campbell defiende su apelación a procedimientos subpersonales en el caso de los modos de presentación demostrativo-perceptuales. Su supuesto es, claro está, que los procedimientos relevantes son aquí los procedimientos subpersonales con los que operan los mecanismos de atención selectiva (diversos "métodos de selección" de determinados "contenidos imaginísticos"). Aplica entonces la línea argumental anterior a estos procedimientos:

[la] atención consciente al objeto no causa meramente [el] uso de estos procedimientos de procesamiento de información. Es también lo que justifica [el] uso de estos procedimientos. Define el objeto de estos procedimientos, cuál es la finalidad de la computación. Y eso es captar el sentido del demostrativo. Si no hubiera conciencia del objeto, se podrían dar esos procedimientos; pero no se captaría (grasp) su objeto [... ]. La respuesta [al tipo de objeción que se mencionó] es que lo que el sujeto capta define el objeto de esos procedimientos subpersonales [...] la comprensión (grasp) que [un sujeto] tiene del demostrativo que está usando define el objeto de los procedimientos subpersonales que subyacen a su uso del demostrativo, incluso aunque el [sujeto] no pueda decir cuáles son esos procedimientos subpersonales. (Campbell 1998, pp. 202-203)

Admitamos por mor del argumento que las consideraciones de Campbell apoyan la tesis de que captar el sentido de una conectiva es necesario para captar el objeto de ciertos procedimientos (en el sentido del fin al que sirven), o que captar un modo de presentación de un objeto material es necesario para captar el objeto de tales 
otros procedimientos de procesamiento de la información (igualmente en el sentido del fin al que sirven). Ello serviría a los objetivos argumentativos de Campbell si a la necesidad unimos la suficiencia. Habría entonces, por decirlo así, una correspondencia uno a uno entre casos de captar o comprender mínimamente el sentido de una conectiva y casos de captar o comprender el objeto de los procedimientos relevantes, e igualmente la habría entre casos de captar un modo demostrativo-perceptual de presentación de un objeto y casos de captar o comprender mínimamente el objeto de los procedimientos correspondientes. Y ésta es justamente la posición a favor de la cual Campbell pretendía argumentar.

Como se ha visto, el argumento de Campbell se basa en la tesis de que captar un sentido o modo de presentación es lo que causa y justifica un determinado conjunto de procedimientos, específicamente relacionados con tal modo de presentación. En el caso del modo de presentación o sentido de una conectiva - que Campbell trata como paradigmático - tales procedimientos son los utilizados "para justificar y hallar las implicaciones de proposiciones que involucran" la conectiva en cuestión (loc. cit.); en el caso de un modo de presentación demostrativo-perceptual los procedimientos relevantes son los "métodos de selección" de las porciones correspondientes de "contenido imaginístico". Su argumento a favor de la tesis se da, como hemos plateado, para el caso presuntamente paradigmático de las conectivas. Pero como el desarrollo de ese argumento deja claro, los procedimientos relacionados con el sentido de las conectivas (por ejemplo, para el caso de la conjunción, derivar "B" de "A y B") son procedimientos de los cuales el sujeto es consciente o al menos puede serlo si se le induce a reflexión. Puede admitirse que, en efecto, la justificación de este tipo de procedimientos está relacionada constitutivamente con la noción de modo de presentación. De manera que la tesis que sirve de base a su argumento podría considerarse justificada si nos referimos al caso de las conectivas o a un caso en el que se dan ese tipo de procedimientos.

Sin embargo, los procedimientos a los que Campbell quiere aplicar su tesis son procedimientos de los que, según el propio Campbell admite, el sujeto no tiene idea alguna ("the subject has no inkling"). Por consiguiente el uso del término "procedimiento" por parte de Campbell es equívoco y esta equivocidad induce la equivocidad en el uso del término "justificación” que utiliza en su argumento. Este término, en efecto, se utiliza en el sentido de justificación racional en la parte de la tesis que constituye la base de su argumento, la parte que podemos considerar justificada, es decir, cuando la tesis se refiere 
al caso de las conectivas, mientras que el hecho de que nuestro sistema perceptual recurra a los procedimientos que Campbell menciona estaría justificado o podría ser considerado correcto, si acaso, en un sentido muy distinto al de la justificación racional; es más bien la fiabilidad de los procedimientos en cuestión lo que estaría en juego, de modo que, en la conclusión, el término "justificación” forzosamente habría de interpretarse — contra las intenciones de Campbell- en un sentido, podríamos decir, puramente fiabilista.

En conclusión, el intento de Campbell de vincular directamente las nociones de una teoría filosófica del contenido mental con nociones de teorías empíricas fracasa. ${ }^{20}$ Por lo tanto, no podemos aceptar las respuestas que, según la propuesta de Campbell, se daría a las preguntas 2 y 3 formuladas en la sección 2 .

Es crucial observar que en la propuesta presentada en la sección anterior no se recurre a nociones de las ciencias cognitivas o las neurociencias. Puede haber razones para pensar que existen procesos en que nuestro sistema cognitivo liga o "combina" propiedades discriminadas en nuestros sistemas sensoriales sin que el sujeto tenga la más mínima conciencia de ello. Tales propiedades - los "rasgos" (features) a los que apelan teorías de la ciencia cognitiva como la de Treisman - pueden incluso estar relacionadas con las rasgos fenoménicos de que se habla de forma generalizada en las descripciones filosóficas de la percepción y también con los qualia que postulan algunas de las teorías filosóficas (como la presentada en la sección 3), pero en cualquier caso responden a sistemas de individuación diferentes: se individúan funcionalmente de acuerdo con las directrices de una teoría científica (cfr. Treisman 1988, 1993). En los términos de la útil distinción a la que hemos recurrido ya: mientras que los rasgos fenoménicos y los qualia son, por supuesto, cosas o propiedades que se postulan en la descripción o explicación de la percepción en el nivel personal, los rasgos a los que se apela en las teorías de la ciencia cognitiva son entidades que conocemos a través de una teoría empírica y el que esos rasgos se combinen o liguen es algo que ocurre en el nivel subpersonal, completamente inaccesible a la introspección y la reflexión filosófica.

Creo que una noción de ligado de rasgos fenoménicos puede hacerse cuando menos plausible en la reflexión filosófica. ${ }^{21}$ En todo caso,

${ }^{20}$ En general, pienso que pretender este tipo de vínculos directos es erróneo. En Fernández Prat 2008 se dilucidan los vínculos entre explicaciones filosóficas y teorías empíricas en el caso de la atención.

${ }^{21}$ Específicamente, sospecho que tal noción es parte constitutiva de nuestros 
no es la primera vez que se postulan operaciones mentales sintéticas sobre una base puramente filosófica. Podemos recordar al respecto la síntesis de la imaginación a la que apeló Kant. ${ }^{22}$ En comparación, lo que aquí se postula parece más modesto: una capacidad de acción muy específicamente delimitada. Y, desde luego, no se pretende postularla sobre la base de algún tipo de filosofía trascendental.

Ciertamente, la propuesta presentada en la sección 4 necesita más clarificación y defensa, presumiblemente en el contexto de una discusión sobre las condiciones de posesión de conceptos demostrativoperceptuales y de conceptos fenoménicos, y también, por otra parte, de la cuestión de la relación entre nociones basadas en la psicología de sentido común informada filosóficamente y nociones de las ciencias cognitivas o la neurociencia. En todo caso, lo dicho aquí sobre la congruencia de los datos sensoriales con el modo de presentación demostrativo-perceptual parece abrir una nueva perspectiva en el esclarecimiento de las relaciones entre el contenido no conceptual y el contenido conceptual de la percepción. ${ }^{23}$

conceptos de rasgos fenoménicos, al menos de los rasgos discernibles en la percepción (o, como mínimo, en último término, de los de la percepción visual). Es decir, poseer el concepto de tales rasgos requeriría, si estoy en lo cierto, no sólo ser consciente de que los mismos se agrupan en espacios (el espacio de las sensaciones de color, por ejemplo), sino también reconocer que pueden aparecer como ligados, pertenecientes a un solo objeto. Esta noción implícita de algún modo en nuestros conceptos de rasgos fenoménicos no pertenece a las ciencias empíricas, no ha sido extraída de ellas, aunque pueda estar relacionada con la noción que aparece en algunas de ellas.

${ }^{22}$ Según la propuesta presentada, con el ligado se efectúa el paso al contenido conceptual ya que el perceptor tiene entonces un modo de presentación demostrativa del objeto y éste se considera como un tipo de contenido conceptual ( $c f r$. nota 15). ¿Es con ello la síntesis el resultado de la aplicación de un concepto, como en la doctrina kantiana? Lo único que puede decirse aquí es que, como en Kant, la síntesis está vinculada a la posesión de un concepto, en este caso el modo de presentación demostrativo. Ahora bien, para determinar si es adecuado considerar este vínculo específicamente como un caso de aplicación, primero habría de determinarse con mayor precisión el carácter de un modo demostrativo. Se habrían de definir las condiciones de posesión de tal concepto, una cuestión objeto de reciente debate que en mi opinión permanece abierta ( $c f r$. Kelly 2001, pp. 403-409 y Speaks 2005, pp. 380-382). A tal fin debería diferenciarse con precisión entre modos demostrativoperceptuales de objetos, y modos demostrativo-perceptuales de propiedades. Quizá entonces podría plantearse, con respecto a los primeros, la cuestión de la relación con un esquema - en el sentido kantiano - de un objeto sensible. Desde luego, no es posible desarrollar aquí ninguna de estas cuestiones ulteriores.

${ }^{23} \mathrm{La}$ investigación correspondiente a este artículo ha recibido financiación del proyecto de investigación HUM2005-07539-C02-02. Agradezco a Daniel Quesada sus múltiples sugerencias y a dos árbitros anónimos sus muy útiles observaciones que han conducido a lo que creo que son grandes mejoras en el artículo. 


\section{BIBLIOGRAFÍA}

Akins, K., 1993, “A Bat without Qualities?”, en M. Davies y G.W. Humphreys (comps.), Consciousness, Blackwell, Oxford.

Block, N., 1995, "On a Confusion about a Function of Consciousness", Behavioral and Brain Sciences, vol. 18, pp. 227-247.

Brewer, B., 1999, Perception and Reason, Oxford University Press, Oxford.

Campbell, J., 2002, Reference and Consciousness, Oxford University Press, Oxford.

__ 1998, "Sense and Consciousness", New Essays on the Philosophy of Michael Dummett, Grazer Philosophiche Studien, vol. 55, Rodopi, Amsterdam, pp. 195-211.

- 1997, "Sense, Reference and Selective Attention", Proceedings of the Aristotelian Society, vol. supl. 71, pp. 55-74.

Chalmers, D., 1996, The Conscious Mind, Oxford University Press, Oxford.

Clark, A., 2000, A Theory of Sentience, Oxford University Press, Oxford.

$\longrightarrow$, 1993, Sensory Qualities, Clarendon Press, Oxford.

Crick, F., 1994, The Astonishing Hypothesis: The Scientific Search for the Soul, Scribner, Nueva York.

Dennett, D., 1988, "Quining Qualia”, reimpreso en W. Lycan (comp.), Mind and Cognition, Oxford University Press, Oxford, 1990. [Versión en castellano: "Quinear los qualia", trad. A.I. Stellino, en Ezcurdia y Hansberg 2003, pp. 213-262.]

— , 1981, "Three Kinds of Intentional Psychology", reimpreso en D. Dennett, 1987, The Intentional Stance, MIT Press, Cambridge, Mass., pp. 37-61.

Dretske, F., 1995, Naturalizing the Mind, MIT Press, Cambridge, Mass.

Dreyfus, H. (comp.), 1982, Husserl, Intentionality, and Cognitive Science, MIT Press, Cambridge, Mass.

Eilan, N., 2001, "Consciousness, Acquaintance and Demonstrative Thought", Philosophy and Phenomenological Research, vol. 63, pp. 433-440.

- 1998, "Perceptual Intentionality, Attention and Consciousness" en O'Hear 1998, pp. 181-202.

Evans, G., 1982, The Varietes of Reference, Oxford University Press, Oxford.

Ezcurdia, M. y O. Hansberg (comps.), 2003, La naturaleza de la experiencia, vol. I, Sensaciones, Instituto de Investigaciones Filosóficas-UnAM, México.

Fernández Prat, O., 2008, "Filosofía y neurociencia: entre la interacción y el eliminativismo", en D. Pérez y L. Fernández Moreno (comps.), Cuestiones filosóficas. Ensayos en honor de Eduardo Rabossi, Catálogos, Buenos Aires, pp. 285-306.

—_ 2006, "Particularity and Reflexivity in the Intentional Content of Perception", Theoria, vol. 56, pp. 133-145.

Føllesdal, D., 1976, "Husserl's Theory of Perception", Ajatus, vol. 36, pp. 95-105. 
Føllesdal, D., 1969, "Husserl's Notion of Noema"; reimpreso en Dreyfus 1982, pp. 73-80.

García-Carpintero, M., 2005, "Intuiciones y contenidos no-conceptuales", en T. Grimaltos y J. Pacho (comps.), La naturalización de la filosofía: Problemas y límites, Pre-Textos, Valencia.

—_, 2003, "Qualia that It Is Right to Quine", Philosophy and Phenomenological Research, vol. 67, pp. 357-377.

_- 2002, "A Vindication of Sense-Data", en C.U. Moulines y K.G. Niebergall (comps.), Argument und Analyse, Mentis, Paderborn, pp. 203-224.

—_, 2001, "Sense Data: The Sensible Approach", Grazer Philosophiche Studien, pp. 18-63.

—, 1999, "Searle on Perception", Teorema, vol. 18, pp. 19-45.

Goodman, N., 1978, Ways of Worldmaking, Hackett, Indianápolis.

Grimaltos, T. y C. Moya, 2006, "Presentation", Theoria, vol. 21, pp. 125132.

Harman, G., 1989, "The Intrinsic Quality of Experience", en J. Tomberlin (comp.), Philosophical Perspectives 4: Philosophy of Mind and Action Theory, Ridgeview, Atascadero, pp. 31-52. [Versión en castellano: "La cualidad intrínseca de la experiencia", trad. A.I. Stellino, en Ezcurdia y Hansberg 2003, pp. 263-287.]

Husserl, E., 1900-1901, Logische Untersuchungen (Husserliana XIX/1 y 2), Nijhoff, La Haya.

—_ 1913, Ideen zu einer reinen Phänomenologie und Phänomenologischen Philosophie, vol. I (Husserliana III), Nijhoff, La Haya. [Versión en castellano: Ideas relativas a una fenomenología pura y una filosofía fenomenológica, Libro Primero, trad. José Gaos, Fondo de Cultura Económica, México, 1949.]

___ 1966, Ideen zu einer reinen Phänomenologie und Phänomenologischen Philosophie, vol. III (Husserliana XI), Nijhoff, La Haya. [Versión en castellano: Ideas relativas a una fenomenología pura y una filosofía fenomenológica, Libro Tercero, trad. L.E. González, Instituto de Investigaciones Filosóficas-UnAM, México, 2000.]

Jackson, F., 1977, Perception: A Representative Theory, Cambridge University Press, Cambridge.

Kelly, S., 2001, "Demonstrative Concepts and Experience", The Philosophical Review, vol. 110, no. 3, pp. 397-420.

Lepore, E. y R. Van Gulick, 1991, John Searle and His Critics, Blackwell, Oxford.

McDowell, J., 1994, Mind and World, Harvard University Press, Cambridge, Mass.

_. 1991, "Intentionality De Re", en Lepore y Van Gulick 1991, pp. 215-225.

- 1982, "Criteria, Defeasibility and Knowledge", Proceedings of the British Academy, vol. 68, pp. 455-479. 
Miller, I., 1984, "Perceptual Reference", Synthese, vol. 61, pp. 35-59.

Mulligan, K., 1995, "Perception", en B. Smith y D.W. Smith (comps.), The Cambridge Companion to Husserl, Cambridge University Press, Cambridge.

O'Hear, A. (comp.), 1998, Contemporary Issues in the Philosophy of Mind, Cambridge University Press, Cambridge.

Peacocke, C., 1998, "Conscious Attitudes, Attention and Self-Knowledge", en C. Wright, B. Smith y C. Macdonald (comps.), Knowing Our Own Minds, Clarendon Press, Oxford, pp. 63-98.

—_ 1992, A Study of Concepts, MIT Press, Cambridge, Mass.

Perkins, M., 1983, Sensing the World, Hackett, Indianapolis.

Pryor, J., 2000, "The Skeptic and the Dogmatist", Noûs, vol. 34, pp. 517549.

Searle, J., 1983, Intentionality: An Essay in the Philosophy of Mind, Cambridge University Press, Cambridge.

Shoemaker, S., 1975, "Phenomenal Similarity", Crítica, vol. 7, pp. 334; reimpreso en S. Shoemaker, 2003, Identity, Cause, and Mind: Philosophical Essays, ed. aum., Clarendon Press, Oxford, pp. 159-183.

Smith, D.W., 1984, "Content and Context of Perception”, Synthese, vol. 61, pp. 61-87.

Smith, D.W. y R. McIntyre, 1982, Husserl and Intentionality: A Study of Mind, Meaning, and Language, Reidel, Dordrecht.

Snowdon, P., 1981, "Perception, Vision and Causation", reimpreso en J. Dancy (comp.), Perceptual Knowledge, Oxford University Press, Oxford, 1988, pp. 192-208.

Speaks, J., 2005, "Is There a Problem about Nonconceptual Content?", Philosophical Review, vol. 114, pp. 359-398.

Treisman, A., 1993, "The Perception of Features and Objets", en A. Baddeley y L. Weiskrantz (comps.), Attention: Selection, Awareness and Control, Clarendon Press, Oxford.

—_ 1988, "Features and Objects: The Fourteenth Bartlett Memorial Lecture", The Quarterly Journal of Experimental Psychology, vol. 40, no. 2, pp. 201-237.

- 1986, "Features and Objects in Visual Processing", Scientific American, vol. 255, no. 5, pp. 106-115.

Treisman, A. y S. Gormican, 1988, "Feature Analysis in Early Vision: Evidence from Search Asymmetries", Psychological Review, vol. 95, pp. 15-48.

Tye, M., 1995, Ten Problems of Consciousness, MIT Press, Cambridge, Mass.

Weiskrantz, L., 1886, Blindsight: A Case Study and Implications, Clarendon Press, Oxford.

Recibido el 20 de julio de 2007; revisado el 15 de julio de 2008; aceptado el 6 de octubre de 2008. 